\title{
Learning to Copy for Automatic Post-Editing
}

\author{
Xuancheng Huang ${ }^{\dagger}$, Yang Liu ${ }^{\dagger+*}$, Huanbo Luan ${ }^{\dagger}$, Jingfang $\mathrm{Xu}^{\S}$ and Maosong Sun ${ }^{\dagger}$ \\ ${ }^{\dagger}$ Institute for Artificial Intelligence \\ State Key Laboratory of Intelligent Technology and Systems \\ Department of Computer Science and Technology, Tsinghua University, Beijing, China \\ Beijing National Research Center for Information Science and Technology \\ ${ }^{\S}$ Sogou Inc., Beijing, China \\ ${ }^{\ddagger}$ Beijing Advanced Innovation Center for Language Resources \\ hxc17@mails.tsinghua.edu.cn, liuyang2011@tsinghua.edu.cn, luanhuanbo@gmail.com, \\ xujingfangesogou-inc.com, smsetsinghua.edu.cn
}

\begin{abstract}
Automatic post-editing (APE), which aims to correct errors in the output of machine translation systems in a post-processing step, is an important task in natural language processing. While recent work has achieved considerable performance gains by using neural networks, how to model the copying mechanism for APE remains a challenge. In this work, we propose a new method for modeling copying for APE. To better identify translation errors, our method learns the representations of source sentences and system outputs in an interactive way. These representations are used to explicitly indicate which words in the system outputs should be copied, which is useful to help CopyNet (Gu et al., 2016) better generate post-edited translations. Experiments on the datasets of the WMT 2016-2017 APE shared tasks show that our approach outperforms all best published results. ${ }^{1}$
\end{abstract}

\section{Introduction}

Automatic post-editing (APE) is an important natural language processing (NLP) task that aims to automatically correct errors made by machine translation systems (Knight and Chander, 1994). It can be considered as an efficient way to modify translations to a specific domain or to incorporate additional information into translations rather than translating from scratch (McKeown et al., 2012; Chatterjee et al., 2015, 2018).

Approaches to APE can be roughly divided into two broad categories: statistical and neural approaches. While early efforts focused on statistical approaches relying on manual feature engineering (Simard et al., 2007; Béchara et al., 2011), neural network based approaches capable of learning representations from data have

\footnotetext{
${ }^{*}$ Corresponding author: Yang Liu

${ }^{1}$ The source code is available at https://github. com/THUNLP-MT/L2COPY 4 APE
}

\begin{tabular}{|l|l|}
\hline$s r c$ & I ate a cake yesterday \\
\hline$m t$ & Ich esse einen Hamburger \\
\hline$p e$ & Ich hatte gestern einen Kuchen gegessen \\
\hline
\end{tabular}

Table 1: Example of automatic post-editing (APE). Given a source sentence $(s r c)$ and a machine translation $(m t)$, the goal of APE is to post-edit the erroneous translation to obtain a correct translation (pe). Our work aims to explicitly model how to copy words from $m t$ to pe (highlighted in bold), which is a common phenomenon in APE.

shown remarkable superiority over their statistical counterparts (Varis and Bojar, 2017; Chatterjee et al., 2017; Junczys-Dowmunt and Grundkiewicz, 2017; Unanue et al., 2018). Most of them cast APE as a multi-source sequence-to-sequence learning problem (Zoph and Knight, 2016): given a source sentence $(s r c)$ and a machine translation $(m t)$, APE outputs a post-edited translation ( $p e$ ).

A common phenomenon in APE is that many words in $m t$ can be copied to pe. As shown in Table 1, two German words "Ich" and "einen" occur in both $m t$ and pe. Note that the positions of copied words in $m t$ and $p e$ are not necessarily identical (e.g., "einen" in Table 1). Our analysis on the datasets of the WMT 2016 and 2017 APE shared tasks shows that over $80 \%$ of words in $m t$ are copied to pe. As APE models not only need to decide which words in $m t$ should be copied correctly, but also should place the copied words in appropriate positions in pe, it is challenging to model copying for APE. Our experiments show that the state-of-the-art APE method (JunczysDowmunt and Grundkiewicz, 2018) only achieves a copying accuracy of $64.63 \%$ (see Table 7).

We believe that existing approaches to APE (Varis and Bojar, 2017; Chatterjee et al., 2017; Junczys-Dowmunt and Grundkiewicz, 2017; Unanue et al., 2018) suffer from two major 


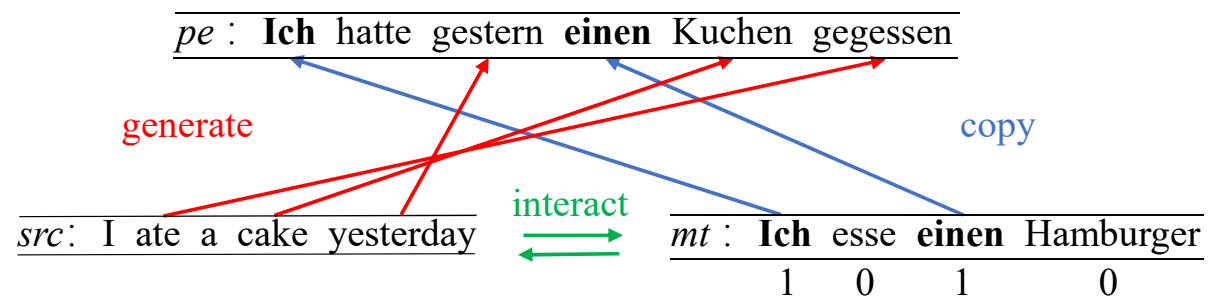

Figure 1: Learning to copy for APE. Our work is based on two key ideas. As both $s r c$ and $m t$ play important roles in APE, the first idea is that $s r c$ and $m t$ should "interact" with each other during representation learning to better generate words from $\operatorname{src}$ and copy words from $m t$ during inference. The second idea is to predict which target words in $m t$ should be copied since it is easy to obtain labeled data automatically by comparing $m t$ and $p e$. The words to be copied (e.g., "Ich") in $m t$ are labeled with 1's and other words (e.g., "esse") with 0's.

drawbacks when modeling the copying mechanism. First, the representations of $s r c$ and $m t$ are learned separately. APE is a two-source sequence-to-sequence learning problem in which both $s r c$ and $m t$ play important roles. On the one hand, if $s r c$ is ignored, it is difficult to identify translation errors related to adequacy in $m t$, especially for fluent but inadequate translations (e.g., $m t$ in Figure 1). On the other hand, $m t$ serves as a major source for generating pe since many words (e.g., "Ich" and "einen" in Figure 1) are copied from $m t$ to $p e$. Intuitively, it is likely to be easier to decide which words in $m t$ should be copied if $s r c$ and $m t$ fully "interact" with each other during representation learning. Although CopyNet (Gu et al., 2016) can be adapted for explicitly modeling the copying mechanism in multi-source sequence-to-sequence learning, the lack of the interaction between $s r c$ and $m t$ still remains a problem.

Second, there is no explicit labeling that indicates which target words in $m t$ should be copied. Existing approaches only rely on the attention between the encoder and decoder to implicitly choose target words to be copied. Given $m t$ and $p e$, it is easy to decide whether a target word in $m t$ should be copied or not. In Figure 1, the words in $m t$ that should be copied are labeled with 1 's. Other words are labeled with with 0 's, which should be re-generated from src. These labels can served as useful supervision signals to help better copy words from $m t$ to $p e$, even when CopyNet is used.

In this work, we propose a new method for modeling the copying mechanism for APE. As shown in Figure 1, our work is based on two key ideas. First, our method is capable of learning the representations of input in an interactive way by enabling $s r c$ and $m t$ to attend to each other during representation learning. This might be useful for deciding when to generate words from $s r c$ and when to copy words from $m t$ during post-editing. Second, it is possible to predict which words in $m t$ should be copied because it is easy to automatically construct labeled data by comparing $m t$ and $p e$. Such predictions can be combined with CopyNet to better model copying for APE. Experiments show that our approach outperforms the best published results on the datasets of the WMT 20162017 APE shared tasks.

\section{Background}

\subsection{Multi-source Sequence-to-Sequence Learning}

Multi-source sequence-to-sequence learning has been widely used in APE in recent years (JunczysDowmunt and Grundkiewicz, 2018; Pal et al., 2018; Tebbifakhr et al., 2018; Shin and Lee, 2018). The architecture of multi-source Transformer is shown in Figure 2(a). It can be equipped with CopyNet (see Section 2.2) to serve as a baseline in our experiments. It is worth noting that $s r c$ and $m t$ are encoded separately.

Let $\mathbf{x}=x_{1} \ldots x_{I}$ be a source sentence (i.e., $s r c$ ) with $I$ words, $\tilde{\mathbf{y}}=\tilde{y}_{1} \ldots \tilde{y}_{K}$ be a translation output by a machine translation system (i.e., $m t$ ) with $K$ words, and $\mathbf{y}=y_{1} \ldots y_{J}$ be the postedited translation (i.e., pe) with $J$ words. The APE model is given by

$$
P(\mathbf{y} \mid \mathbf{x}, \tilde{\mathbf{y}} ; \boldsymbol{\theta})=\prod_{j=1}^{J} P\left(y_{j} \mid \mathbf{x}, \tilde{\mathbf{y}}, \mathbf{y}_{<j} ; \boldsymbol{\theta}\right),
$$

where $y_{j}$ is the $j$-th target word in $p e, \mathbf{y}_{<j}=$ $y_{1} \ldots y_{j-1}$ is a partial translation, $\boldsymbol{\theta}$ is a set of model parameters, and $P\left(y_{j} \mid \mathbf{x}, \tilde{\mathbf{y}}, \mathbf{y}<j ; \boldsymbol{\theta}\right)$ is a word-level translation probability. 


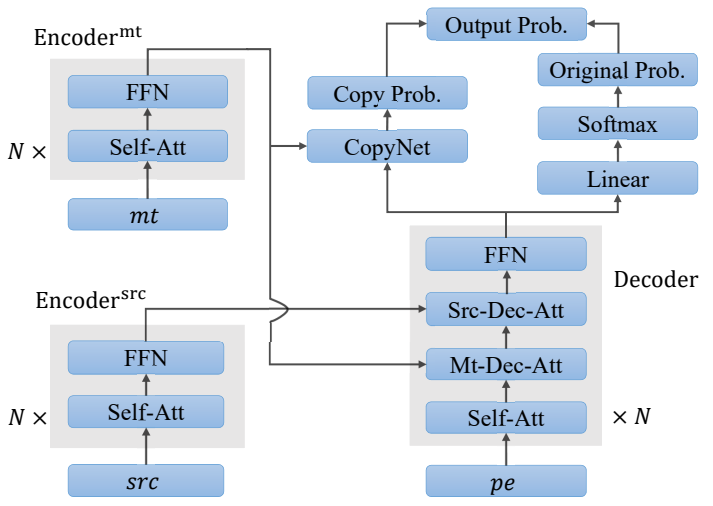

(a)

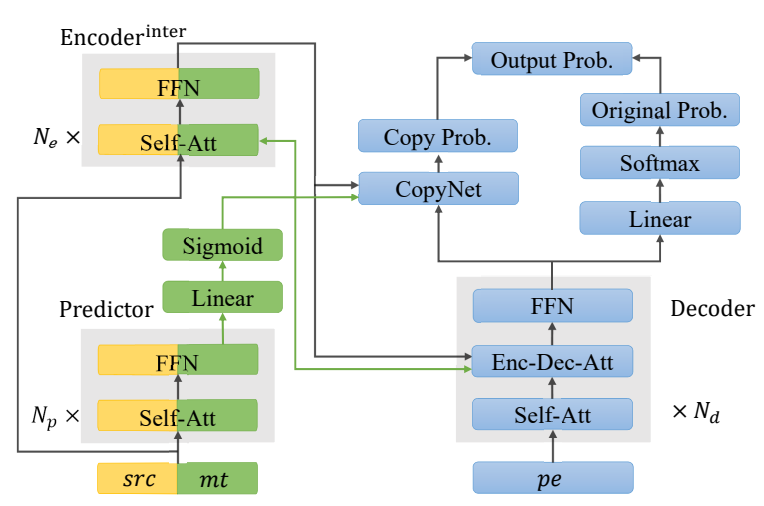

(b)

Figure 2: (a) The architecture of multi-source Transformer (Junczys-Dowmunt and Grundkiewicz, 2018) equipped with CopyNet ( $\mathrm{Gu}$ et al., 2016) and (b) the architecture of our approach. While the existing work learns the representations of $s r c$ and $m t$ separately, our approach allows for learning the representations of $s r c$ and $m t$ in an interactive way by concatenating them as a single input. In addition, our approach introduces a Predictor module to explicitly indicate which words in $m t$ should be copied.

The word-level translation probability in Eq. (1) is computed as

$$
\begin{aligned}
& \mathbf{H}^{\mathrm{src}}=\text { Encoder }^{\mathrm{src}}(\mathbf{x}, \boldsymbol{\theta}), \\
& \mathbf{H}^{\mathrm{mt}}=\operatorname{Encoder}^{\mathrm{mt}}(\tilde{\mathbf{y}}, \boldsymbol{\theta}), \\
& \mathbf{h}_{j}^{\mathrm{pe}}=\operatorname{Decoder}\left(\mathbf{y}_{<j}, \mathbf{H}^{\mathrm{src}}, \mathbf{H}^{\mathrm{mt}}, \boldsymbol{\theta}\right), \\
& P\left(y_{j} \mid \mathbf{x}, \tilde{\mathbf{y}}, \mathbf{y}_{<j} ; \boldsymbol{\theta}\right) \propto \exp \left(\mathbf{h}_{j}^{\mathrm{pe}} \mathbf{W}_{g}\right),
\end{aligned}
$$

where Encoder ${ }^{\mathrm{src}}(\cdot)$ is the encoder for src, $\mathbf{H}^{\text {src }}$ is the real-valued representation of $s r c$, Encoder $^{\mathrm{mt}}(\cdot)$ is the encoder for $m t, \mathbf{H}^{\mathrm{mt}}$ is the representation of $m t$, Decoder $(\cdot)$ is the decoder, $\mathbf{h}_{j}^{\text {pe }}$ is the representation of the $j$-th target word $y_{j}$ in pe. $\mathbf{W}_{g} \in \mathbb{R}^{d \times \mathcal{V}_{y}}$ is a weight matrix, $d$ is the dimension of hidden states, and $\mathcal{V}_{y}$ is the target vocabulary size.

A limitation of the aforementioned model is that $s r c$ and $m t$ are encoded separately without interacting with each other, which might lead to the inability to find which $s r c$ word is untranslated and which $m t$ word is incorrect. For example, the $m t$ sentence in Figure 1 is fluent and meaningful. Without $s r c$, the APE system is unable to identify translation errors. In addition, the multisource Transformer does not explicitly model the copying between $m t$ and $p e$ in neither the Encoder nor the Decoder.

\subsection{CopyNet}

CopyNet (Gu et al., 2016) is a widely used method for modeling copying in sequence-to-sequence learning. It has been successfully applied to single-turn dialogue (Gu et al., 2016), text sum- marization (See et al., 2017), and grammar error correction (Zhao et al., 2019).

It is possible to extend the multi-source Transformer with CopyNet to explicitly model the copying mechanism, as shown in Figure 2(a). CopyNet defines the word-level translation probability in Eq. (1) as a linear interpolation of copying and generating probabilities:

$$
\begin{aligned}
P\left(y_{j} \mid \mathbf{x}, \tilde{\mathbf{y}}, \mathbf{y}_{<j} ; \boldsymbol{\theta}\right)= & \gamma_{j} \times P^{\text {copy }}\left(y_{j}\right) \\
& +\left(1-\gamma_{j}\right) \times P^{\text {gen }}\left(y_{j}\right),
\end{aligned}
$$

where $P^{\text {copy }}\left(y_{j}\right)$ is the copying probability for $y_{j}$, $P^{\text {gen }}\left(y_{j}\right)$ is the generating probability for $y_{j}$, and $\gamma_{j}$ is a gating weight. They are defined as follows:

$$
\begin{aligned}
P^{\text {copy }}\left(y_{j}\right) & \propto \exp \left(g\left(\mathbf{H}^{\mathrm{mt}}, \mathbf{h}_{j}^{\mathrm{pe}}\right)\right), \\
P^{\mathrm{gen}}\left(y_{j}\right) & \propto \exp \left(\mathbf{h}_{j}^{\mathrm{pe}} \mathbf{W}_{g}\right), \\
\gamma_{j} & =u\left(\mathbf{H}^{\mathrm{mt}}, \mathbf{h}_{j}^{\mathrm{pe}}\right),
\end{aligned}
$$

where $g(\cdot)$ and $u(\cdot)$ are non-linear functions. See (Zhao et al., 2019) for more details.

Copying in APE involves two kinds of decisions: (1) choosing words in $m t$ to be copied and (2) placing the copied words in appropriate positions in pe. CopyNet makes the two kinds of decisions simultaneously. We conjecture that if which words in $m t$ should be copied can be explicitly indicated, it might be easier for CopyNet to copy words from $m t$ to pe correctly. Therefore, it is necessary to design a new method for identifying words to be copied. 


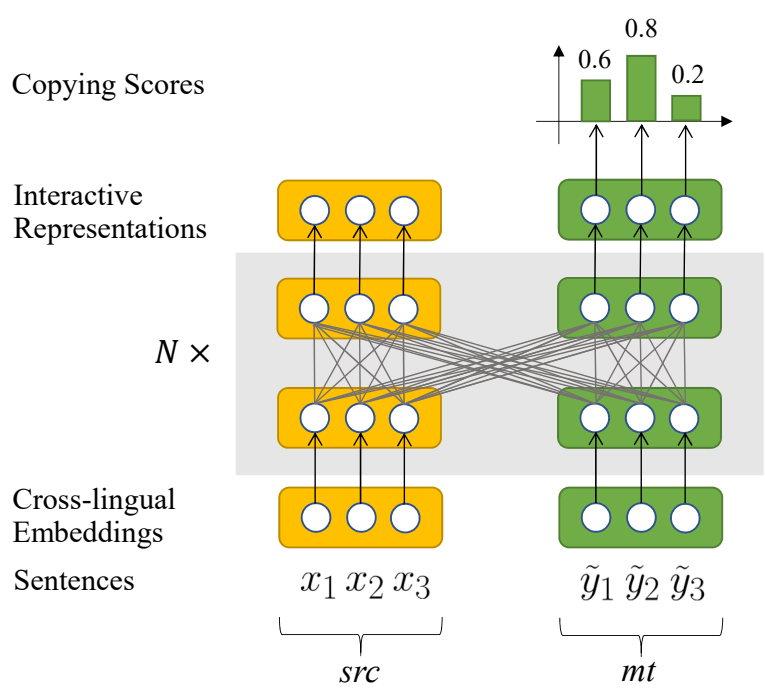

Figure 3: Interactive representation learning. Concatenated to serve as a single input, $s r c$ and $m t$ attend to each other during representation learning. Note that learnable weights of $s r c$ and $m t$ are shared. Interactive representation learning is used in both Predictor and Encoder. For example, copying scores are predicted based on the learned representations (see Eq. (13)).

\section{Approach}

Figure 2(b) shows the overall architecture of our approach. It differs from previous work in two aspects. First, we propose to let $\operatorname{src}$ and $m t$ "interact" with each other to learn better representations (Section 3.1). Second, our approach introduces a Predictor module to predict words to be copied (Section 3.2). Section 3.3 describes how to train our APE model.

\subsection{Interactive Representation Learning}

We propose an interactive representation learning method by making $s r c$ and $m t$ attend to each other. Following Lample and Conneau (2019) and He et al. (2018), we concatenate $s r c$ and $m t$ in the dimension of sentence length with additional position and language embeddings:

$$
\begin{aligned}
\mathbf{X}_{i} & =\mathbf{E}^{\text {token }}\left[x_{i}\right]+\mathbf{E}^{\text {pos }}[i]+\mathbf{E}^{\text {lang }}[0], \\
\tilde{\mathbf{Y}}_{k} & =\mathbf{E}^{\text {token }}\left[\tilde{y}_{k}\right]+\mathbf{E}^{\text {pos }}[k]+\mathbf{E}^{\text {lang }}[1],
\end{aligned}
$$

where $\mathbf{X}_{i}$ is the embedding of the $i$-th source word $x_{i}, \tilde{\mathbf{Y}}_{k}$ is the embedding of the $k$-th target word $\tilde{y}_{k}$, and $\mathbf{E}^{\text {token }}, \mathbf{E}^{\text {pos }}$, and $\mathbf{E}^{\text {lang }}$ are the token, position and language embedding matrices.

As shown in Figure 3, the representation of src and $m t$ can be learned jointly:

$$
\mathbf{H}^{\text {inter }}=\text { Encoder }^{\text {inter }}([\mathbf{X} ; \tilde{\mathbf{Y}}], \boldsymbol{\theta}),
$$

where Encoder ${ }^{\text {inter }}(\cdot)$ is the interactive Encoder and $[\mathbf{X} ; \tilde{\mathbf{Y}}]$ is the concatenation of $\mathbf{X}$ and $\tilde{\mathbf{Y}}$ in the dimension of sentence length.

As shown in Figure 2(b), the multi-source Encoders are replaced by the interactive Encoder, which enables $s r c$ and $m t$ to attend to each other. ${ }^{2}$ We expect that enabling the interactions between them can help to strengthen the ability of the model to find which words in $s r c$ is untranslated and which words in $m t$ is correct. Note that interactive representation learning is used both in Predictor and Encoder. In the following, we will describe how to predict which words in $m t$ should be copied based on these learned representations.

\subsection{Predicting Words to be Copied}

Given $m t$ and $p e$, we can label each word in $m t$ as 0 or 1 . We use 1 to denote that the word is to be copied (e.g. "Ich" and "einen" in Figure 1) and 0 not to be copied (e.g. "esse" and "Hamburger" in Figure 1). It is possible to use the Longest Common Sequence (LCS) (Wagner and Fischer, 1974) algorithm to obtain common sequences between $m t$ and $p e$. If the word in $m t$ also appears in the common sequences, it will be labeled 1; otherwise, it will be labeled 0 . We denote these labels as $l_{1} \ldots l_{K}$.

We propose a Predictor module to predict words to be copied. As discrete labels are nondifferentiable during training, the Predictor module outputs copying scores instead for the target words in $m t$ :

$$
\mathbf{s}=\operatorname{sigmoid}\left(\left[\mathbf{H}_{I+1}^{\mathrm{pred}} ; \cdots ; \mathbf{H}_{I+K}^{\mathrm{pred}}\right] \mathbf{W}_{s}\right),
$$

where $\mathbf{s} \in \mathbb{R}^{K \times 1}$ is a vector of copying scores corresponding to the $K$ words in $m t, \mathbf{H}^{\text {pred }} \in$ $\mathbb{R}^{(I+K) \times d}$ is the representation of $s r c$ and $m t$ :

$$
\mathbf{H}^{\text {pred }}=\operatorname{Predictor}([\mathbf{X} ; \tilde{\mathbf{Y}}] ; \boldsymbol{\theta}),
$$

and $\mathbf{W}_{s} \in \mathbb{R}^{d \times 1}$ is a weight matrix. Only the representation of $m t$ (i.e., $\left[\mathbf{H}_{I+1}^{\text {pred }} ; \cdots ; \mathbf{H}_{I+K}^{\text {pred }}\right]$ ) is used for calculating copying scores. ${ }^{3}$

As shown in Figure 2(b), copying scores can be incorporated into three parts of our model: the

\footnotetext{
${ }^{2}$ Note that the Predictor and Encoder do not share their weights because we found that sharing leads to degraded performance in experiments.

${ }^{3}$ It is also possible to predict which source words in $s r c$ should be used to generate non-copied words in pe (e.g., "Kuchen" in Figure 1). This can be done by generating explicit labels using bilingual word alignment. We leave this for future work.
} 


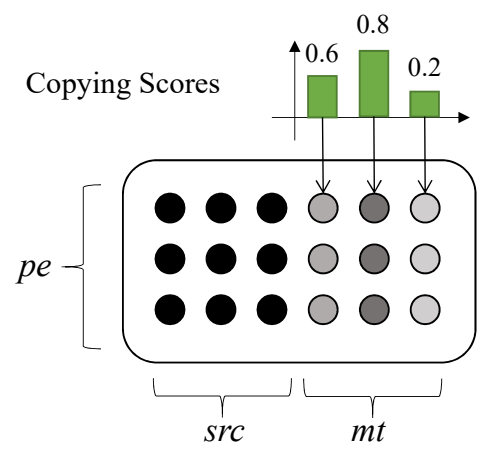

Figure 4: Copying scores as scaling masks. The copying scores are used to modify the attention layers of the Encoder, Decoder, and CopyNet.

Encoder, the Decoder, and the CopyNet. Inspired by Yang et al. (2018)'s strategy to integrate localness to self-attention, we propose to incorporate copying scores into our model by modifying attention weights involved in the aforementioned three modules.

The original scaled dot-product attention (Vaswani et al., 2017) is defined as

$$
\begin{aligned}
\text { energy } & =\frac{\mathbf{q} \mathbf{K}^{\top}}{\sqrt{d}}, \\
\operatorname{Att}(\mathbf{q}, \mathbf{K}) & =\operatorname{softmax}(\text { energy }),
\end{aligned}
$$

where $\mathbf{q} \in \mathbb{R}^{1 \times d}$ is the query vector, $\mathbf{K} \in$ $\mathbb{R}^{(I+K) \times d}$ is the key matrix, and energy $\in$ $\mathbb{R}^{1 \times(I+K)}$ is the "energy" vector.

As shown in Figure 4, the copying scores can be used to form a scaling mask on the attention sub-layer: ${ }^{4}$

$$
\operatorname{Att}(\mathbf{q}, \mathbf{K})=\operatorname{softmax}\left(\text { energy } \odot[\mathbf{m} ; \mathbf{s}]^{\top}\right)
$$

where $\mathbf{m}=\{1.0\}^{I}$ is a masking vector corresponding to $\operatorname{src}$ and $\mathbf{s} \in \mathbb{R}^{K \times 1}$ is the vector of copying scores calculated by Eq. (13) corresponding to $m t$. Note that copying scores are used to only change the attention weights related to $m t$ while the $\operatorname{src}$ part is unchanged.

\subsection{Training}

The training objective of our approach $L_{\text {all }}(\boldsymbol{\theta})$ consists of three parts:

$$
\begin{aligned}
L_{\text {all }}(\boldsymbol{\theta})= & (1-\alpha) \times\left(L_{\text {ape }}(\boldsymbol{\theta})+\lambda \times L_{\text {copy }}(\boldsymbol{\theta})\right) \\
& +\alpha \times L_{\text {pred }}(\boldsymbol{\theta})
\end{aligned}
$$

\footnotetext{
${ }^{4}$ Actually, we let "energy" vector minus its minimum value to keep it non-negative.
}

where $\alpha$ and $\lambda$ are hyper-parameters.

The first part is the original log-likelihood loss function of APE: ${ }^{5}$

$$
L_{\text {ape }}(\boldsymbol{\theta})=-\log P(\mathbf{y} \mid \mathbf{x}, \tilde{\mathbf{y}} ; \boldsymbol{\theta}) .
$$

The second part is related to the CopyNet:

$$
L_{\text {copy }}(\boldsymbol{\theta})=\frac{1}{K} \sum_{k=1}^{K}\left(l_{k}-c_{k}\right)^{2},
$$

where $l_{k}$ is the ground-truth label (see Section 3.2) and $c_{k}$ is a quantity that measures how likely the $k$-th word in $m t$ to be copied by CopyNet:

$$
c_{k}=\sum_{j=1}^{J} \gamma_{j} \times P^{\text {copy }}\left(\tilde{y}_{k}\right) .
$$

Note that $\gamma \times P^{\text {copy }}(y)$ is the term related to copying the target word $y$ in Eq. (6).

The third part is a cross-entropy loss related to the Predictor:

$$
\begin{aligned}
L_{\text {pred }}(\boldsymbol{\theta})=-\sum_{k=1}^{K} & {\left[l_{k} \log \left(s_{k}\right)\right.} \\
& \left.+\left(1-l_{k}\right) \log \left(1-s_{k}\right)\right],
\end{aligned}
$$

where $s_{k}$ is the copying score of the $k$-th word $\tilde{y}_{k}$ in $m t$.

Finally, we use an optimizer to find the model parameters that minimize the overall loss function:

$$
\hat{\boldsymbol{\theta}}=\underset{\boldsymbol{\theta}}{\operatorname{argmin}}\left\{L_{\text {all }}(\boldsymbol{\theta})\right\} .
$$

\section{Experiments}

\subsection{Setup}

\section{Datasets}

We evaluated our approach on the WMT APE datasets, which often distinguish between two tasks: phrase-based statistical machine translation (i.e., PBSMT) and neural machine translation (i.e., NMT). All these APE datasets consist of EnglishGerman triplets containing source text $(s r c)$, the translations ( $m t$ ) from a "black-box" MT system and the corresponding human-post-edits ( $p e$ ). The statistics for the WMT APE datasets are shown in Table 2. In addition to the official dataset, the organizers also recommend using additional datasets

\footnotetext{
${ }^{5}$ For simplicity, we use sentence-level loss functions here. In practice, we use corpus-level loss functions.
} 


\begin{tabular}{|c|l|r|}
\hline Category & Dataset & \# Sent. \\
\hline \hline \multirow{4}{*}{ PBSMT } & training set & 23,000 \\
& dev2016 & 1,000 \\
& test2016 & 2,000 \\
& test2017 & 2,000 \\
\hline \multirow{2}{*}{ NMT } & training set & 13,442 \\
& dev2018 & 1,000 \\
\hline \multirow{5}{*}{ Additional } & artificial-small & 526,368 \\
& artificial-big & $4,391,180$ \\
& eSCAPE-PBSMT & $7,258,533$ \\
& eSCAPE-NMT & $7,258,533$ \\
\hline
\end{tabular}

Table 2: Statistics of the English-German datasets in the WMT APE task. Note that the NMT official data only contains training and development sets.

(Junczys-Dowmunt and Grundkiewicz, 2016; Negri et al., 2014).

We used the WMT official dataset for the PBSMT task and the NMT task separately. The artificial training data (Junczys-Dowmunt and Grundkiewicz, 2016) was also used for both tasks. More precisely, we used the concatenation of the official training data and the artificial-small data to learn a truecasing model (Koehn et al., 2007) and obtain sub-word units using byte-pair encoding (BPE) (Sennrich et al., 2015) with 32k merges. Then, we applied truecasing and BPE to all datasets. We oversampled the official training data 20 times and concatenated them with both artificial-small and artificial-big datasets (JunczysDowmunt and Grundkiewicz, 2018). Finally, we obtained a dataset containing nearly $5 \mathrm{M}$ triplets for both tasks. To test our approach on a larger PBSMT dataset, we used the eSCAPE synthetic dataset (Negri et al., 2014), which contains 7.2M sentences. By including the eSCAPE dataset, the training set is enlarged to nearly $12 \mathrm{M}$ sentences.

\section{Hyper-Parameter Settings}

For the original Transformer model, CopyNet and our approach, the hidden size was set to 512 and the filter size was set to 2,048 . The number of individual attention heads was set to 8 for multi-head attention. We set $N=N_{e}=N_{d}=6, N_{p}=3$ and we tied all three src, $m$ t, pe embeddings for saving memory. The embeddings and softmax weights were also tied. In training, we used Adam (Kingma and Ba, 2014) for optimization. Each mini-batch contains approximately $25 \mathrm{~K}$ tokens. We used the learning rate decay policy described

\begin{tabular}{|cc|cc|}
\hline$\alpha$ & $\lambda$ & TER $\downarrow$ & BLEU $\uparrow$ \\
\hline \hline 0.1 & 1.0 & 18.83 & 72.59 \\
0.5 & 1.0 & 18.45 & 72.83 \\
0.9 & 0.1 & 18.89 & 72.27 \\
0.9 & 0.5 & 18.47 & 72.83 \\
0.9 & 1.0 & $\mathbf{1 8 . 3 8}$ & $\mathbf{7 2 . 9 9}$ \\
\hline
\end{tabular}

Table 3: Effect of $\alpha$ and $\lambda$. The TER and BLEU scores are calculated on the WMT 2016 APE official development set.

by (Vaswani et al., 2017). In decoding, the beam size was set to 4 . We used the length penalty (Wu et al., 2016) and set the hyper-parameter to 1.0. The other hyper-parameter settings were the same as the Transformer model (Vaswani et al., 2017). We implemented our approach on top of the opensource toolkit THUMT (Zhang et al., 2017). ${ }^{6}$

\section{Evaluation Metrics}

We used the same evaluation metrics as the official WMT APE task (Chatterjee et al., 2018): casesensitive BLEU and TER. BLEU is computed by multi-bleu.perl (Koehn et al., 2007). TER is calculated using TERcom. ${ }^{7}$

\section{Baselines}

We compared our approach with the following seven baselines:

1. ORIGINAL: the original $m t$ without any postediting.

2. Copynet (Gu et al., 2016; Zhao et al., 2019): the multi-source Transformer equipped with CopyNet (see Figure 2(a)).

3. NPI-APE (Vu and Haffari, 2018): a neural programmer-interpreter approach.

4. MS_UEDIN (Junczys-Dowmunt and Grundkiewicz, 2018): a multi-source Transformerbased APE system that shares the encoders of $s r c$ and $m t$. It is the champion of the WMT 2018 APE shared task.

5. UsAAR_DFKI (Pal et al., 2018): a multisource Transformer-based APE system with a joint encoder that attends over a combination of two encoded sequences. It is a participant of the WMT 2018 APE shared task.

\footnotetext{
${ }^{6}$ https://github.com/THUNLP-MT/THUMT

${ }^{7}$ http://www.cs.umd.edu/snover/tercom/
} 


\begin{tabular}{|c|c|c|c|c|c|c|}
\hline \multirow{2}{*}{ System } & \multicolumn{2}{|c|}{ TEST16 } & \multicolumn{2}{|c|}{ TEST17 } & \multicolumn{2}{|c|}{ TEST16+17 } \\
\hline & TER $\downarrow$ & $\mathrm{BLEU} \uparrow$ & TER $\downarrow$ & BLEU $\uparrow$ & TER $\downarrow$ & $\mathrm{BLEU} \uparrow$ \\
\hline (1) ORIGINAL & 24.76 & 62.11 & 24.48 & 62.49 & 24.62 & 62.30 \\
\hline \multicolumn{7}{|c|}{ WMT 2017 official $+\operatorname{artificial}(5 \mathrm{M})$} \\
\hline (2) NPI-APE & 22.07 & 66.67 & 22.58 & 65.52 & - & - \\
\hline (3) PosteCH & 19.14 & 70.98 & 19.26 & 70.50 & - & - \\
\hline (4) FBK & 18.79 & 71.48 & 19.54 & 70.09 & - & - \\
\hline (5) MS_UEDIN ensemble $_{\text {. }}$ & 18.86 & 71.04 & 19.03 & 70.46 & - & - \\
\hline (6) COPYNET & 18.91 & 71.64 & 19.47 & 70.75 & 19.19 & 71.19 \\
\hline (7) Ours & 18.39 & 72.50 & 18.81 & 71.35 & 18.60 & 71.92 \\
\hline (8) Ours ensemble $_{\text {. }}$ & 17.77 & 73.19 & 18.41 & 72.09 & 18.09 & 72.62 \\
\hline \multicolumn{7}{|c|}{ WMT 2017 official + artificial + eSCAPE (12M) } \\
\hline (9) USAAR_DFKI & - & 68.52 & - & 68.91 & - & - \\
\hline (10) Ms_UEDIN ensemble & 17.34 & 73.43 & 17.47 & 72.84 & - & - \\
\hline (11) COPYNET & 18.06 & 72.77 & 18.29 & 71.89 & 18.18 & 72.37 \\
\hline (12) Ours & 17.45 & 73.51 & 17.77 & 72.98 & 17.61 & 73.24 \\
\hline (13) Ours ensemble & 17.06 & 74.00 & 17.37 & 73.26 & $\mathbf{1 7 . 2 2}$ & 73.62 \\
\hline
\end{tabular}

Table 4: Results on the English-German PBSMT sub-task. "TEST16+17" is the concatenation of "TEST16" and "TEST17". MS_UEDIN ensemble $_{\text {and Ours }}$ ensemble used ensembles of four models.

6. Postech (Shin and Lee, 2018): a multisource Transformer-based APE system with two encoders. It is a participant of the WMT 2018 APE shared task.

7. FBK (Tebbifakhr et al., 2018): a multi-source Transformer-based APE system with two encoders. It is a participant of the WMT 2018 APE shared task.

We implemented COPYNET also on top of THUMT (Zhang et al., 2017). The results of all other baselines were taken from the corresponding original papers.

\subsection{Effect of Hyper-parameters}

We first investigated the effect of the hyperparameters $\alpha$ and $\lambda$ in Eq. (18). As shown in Table 3 , using $\alpha=0.9$ and $\lambda=1.0$ achieves the best performance in terms of TER and BLEU on the WMT 2016 development set, suggesting that both the Predictor and CopyNet play important roles in our approach. Therefore, we set $\alpha=0.9$ and $\lambda=1.0$ in the following experiments.

\subsection{Main Results}

\section{Results on the PBSMT Sub-task}

Table 4 shows the results of the PBSMT sub-task. We used the development set of the WMT 2016

\begin{tabular}{|l|cc|}
\hline System & TER $\downarrow$ & BLEU $\uparrow$ \\
\hline \hline ORIGINAL & 15.08 & 76.76 \\
\hline POSTECH & 14.94 & 77.26 \\
\hline COPYNET & 15.12 & 77.05 \\
\hline Ours & $\mathbf{1 4 . 8 8}$ & $\mathbf{7 7 . 4 0}$ \\
\hline
\end{tabular}

Table 5: Experiments on the WMT 2018 EnglishGerman NMT sub-task. The TER and BLEU scores are calculated on the WMT 2018 APE NMT sub-task development set.

APE PBSMT sub-task for model selection for our approach.

Under the small-data training condition (i.e., 5M), our single model (i.e., System 7) outperforms all single-model baselines on all test sets. The superiority over COPYNET (i.e., System 6) suggests that interactive representation learning and incorporating copying scores are effective in improving APE. Our approach that uses the ensemble of four models (i.e., System 8 ) also improves over the best published result (i.e., System 5).

Under the large-data training condition (i.e., 12M), we find that our single (i.e., System 12) and ensemble (i.e., System 13) models still outperform the best single (i.e., System 11) and ensemble (i.e., System 10) models of baselines. 


\begin{tabular}{|c|cccc||cc|cc|}
\hline \multirow{2}{*}{ ID } & \multicolumn{5}{|c||}{ Module } & \multicolumn{2}{c|}{ DEV16 } & \multicolumn{2}{c|}{ TEST16+17 } \\
\cline { 2 - 9 } & Interactive & Predictor & CopyNet & Joint Training & TER $\downarrow$ & BLEU $\uparrow$ & TER $\downarrow$ & BLEU $\uparrow$ \\
\hline \hline 1 & $\sqrt{ }$ & $\times$ & $\times$ & $\times$ & 18.74 & 72.21 & 19.11 & 71.03 \\
2 & $\times$ & $\times$ & $\sqrt{ }$ & $\times$ & 19.33 & 71.86 & 19.19 & 71.19 \\
3 & $\times$ & $\sqrt{ }$ & $\sqrt{ }$ & $\sqrt{ }$ & 19.11 & 72.03 & 19.07 & 71.30 \\
4 & $\sqrt{ }$ & $\times$ & $\sqrt{ }$ & $\times$ & 18.62 & 72.42 & 18.91 & 71.53 \\
5 & $\sqrt{ }$ & $\sqrt{ }$ & $\times$ & $\times$ & 18.53 & 72.53 & 18.85 & 71.54 \\
6 & $\sqrt{ }$ & $\sqrt{ }$ & $\sqrt{ }$ & $\times$ & 18.44 & 72.96 & 18.77 & 71.75 \\
\hline 7 & $\sqrt{ }$ & $\sqrt{ }$ & $\sqrt{ }$ & $\sqrt{ }$ & $\mathbf{1 8 . 3 8}$ & $\mathbf{7 2 . 9 9}$ & $\mathbf{1 8 . 6 0}$ & $\mathbf{7 1 . 9 2}$ \\
\hline
\end{tabular}

Table 6: Ablation study. "Interactive" denotes interactive representation learning (Section 3.1), "Predictor" denotes detecting correct words (Section 3.2), "CopyNet" denotes the CopyNet used in our approach (Section 2.2), and "Joint Training" denotes the combination of three loss functions (Section 3.3).

\section{Results on the NMT Sub-task}

Table 5 shows the results on the NMT sub-task. Besides ORIGINAL and COPYNET, we also compared our approach with POSTECH (Shin and Lee, 2018), which is the only participating system that released the results on the development set of the WMT 2018 NMT sub-task. ${ }^{8}$ We find that our approach also outperforms all baselines.

\subsection{Ablation Study}

Table 6 shows the results of ablation study. It is clear that interactive representation learning plays a critical role since removing it impairs postediting performance (line 3). As shown in line 4, the Predictor is also an essential part of our approach. CopyNet and joint training are also shown to be beneficial for improving APE (lines 5 and 6) but seem to have relatively smaller contributions than interactive representation learning and predicting words to be copied.

\subsection{Results on Prediction Accuracy}

The Predictor is an important module in our approach as it predicts which words in $m t$ should be copied. Given the ground-truth labels, it is easy to calculate prediction accuracy by casting the prediction as a binary classification problem. We find that the Predictor achieves a prediction accuracy of $85.09 \%$ on the development set.

\subsection{Comparison of Copying Accuracies}

A target word $y$ in a machine translation $\tilde{\mathbf{y}}$ is called to be correctly copied to an automatic edited translation $\hat{\mathbf{y}}$ if and only if the positions where $y$ occurs

\footnotetext{
${ }^{8}$ As POSTECH only used small data for training, the eSCAPE datasets were not used in this experiment for a fair comparison.
}

\begin{tabular}{|l|c|}
\hline System & Accuracy \\
\hline \hline MS_UEDIN & 64.63 \\
COPYNET & 64.72 \\
\hline Ours & $\mathbf{6 5 . 6 1}$ \\
\hline
\end{tabular}

Table 7: Comparison of copying accuracies.

in $\hat{\mathbf{y}}$ and $\mathbf{y}$ (i.e., the ground-truth edited translation) are identical. Therefore, it is easy to define copying accuracy to measure how well the copying mechanism works.

Table 7 shows the comparison of copying accuracies between Ms_UEDIN, COPYNET, and our approach. We find that our approach outperforms the two baselines. However, the copying accuracy of our approach is almost $20 \%$ lower than the prediction accuracy (i.e., $65.61 \%$ vs. $85.09 \%$ ), indicating that it is much more challenging to place the copied words in correct positions.

\subsection{Visualization}

Figure 5 gives an example that illustrates how copying scores influence attention. It shows the heatmap of the Enc-Dec-Attention, which averages over 8 different heads. Only the attention weights beween $p e$ and $m t$ are included. We take the second layer of Enc-Dec-Attention for example. The $\mathrm{x}$-axis represents $m t$ and the y-axis represents pe. The darker the color, the higher the copying scores.

We find that words "mit" and "dem" are identified by the Predictor. Accordingly, the attention weights corresponding to these words are decreased since the columns corresponding to these words have lighter color. As a result, all words in $m t$ other than "mit" and "dem" are copied to $p e$. 


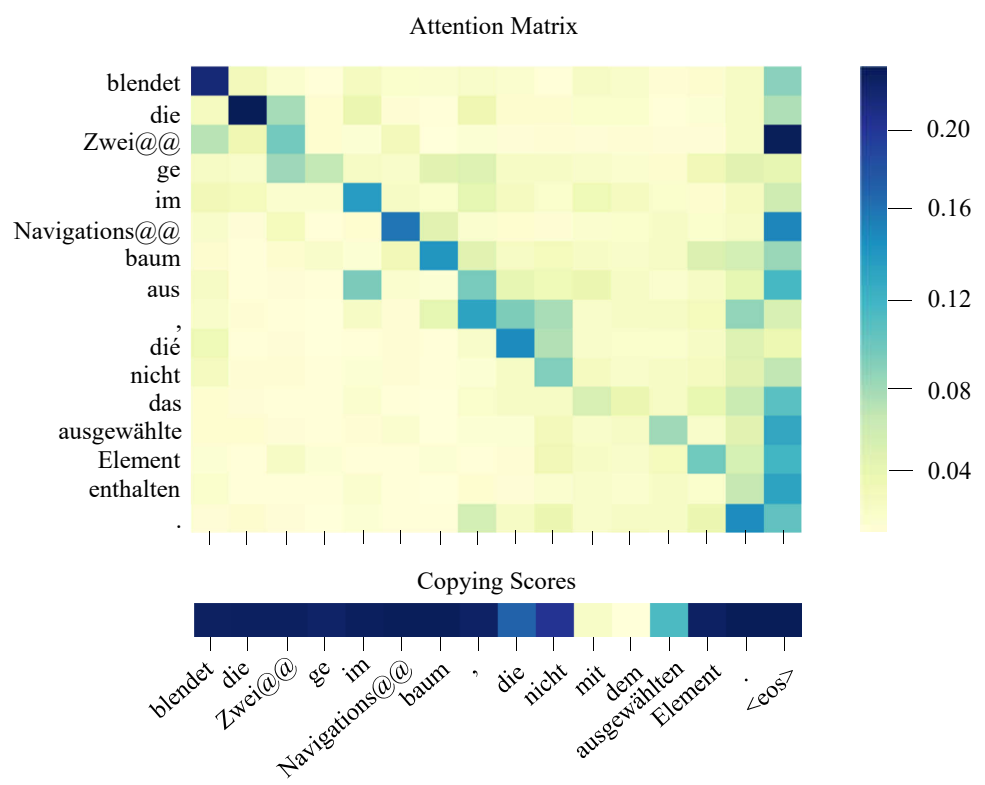

Figure 5: Example of the heatmap of attention and copying scores. The x-axis is $m t$ and the y-axis is pe. The Predictor successfully detects the incorrect word "mit" and "dem" and gives low copying scores to these words and then decrease the importance of them in attention. Other words in $m t$ are correctly copied to pe.

\section{Related Work}

\subsection{Multi-source Sequence-to-Sequence Learning}

Recently, multi-source Transformer-based APE systems (Junczys-Dowmunt and Grundkiewicz, 2018; Pal et al., 2018; Tebbifakhr et al., 2018; Shin and Lee, 2018) have achieved the state-of-the-art results on the datasets of the WMT APE shared task. Our work differs from prior studies by enabling interactions between src and $m t$ and explicitly detecting words to be copied.

\subsection{The Copying Mechanism}

Zhao et al. (2019) apply CopyNet (Gu et al., 2016) to grammar error correction. Their approach generates labels similar to ours, but only uses them to perform mutli-task learning. Libovický et al. (2016) first introduce CopyNet to APE but do not provide a detailed description of their method and experimental results. We show that interactive representation learning and explicit indication of words are important for modeling copying in APE.

\subsection{Interactive Representation Learning}

Niehues et al. (2016) simply concatenate the output of the PBSMT system and the source sentence to serve as the input the NMT system without enabling multi-layer interactive learning. Lample and Conneau (2019) used cross-lingual setting to enable cross-lingual language model pre-training. We propose to let $s r c$ and $m t$ fully interact with each other to make it easier to decide which words in $m t$ should be copied.

\section{Conclusion}

We have presented a new method for modeling the copying mechanism for automatic post-editing. By making the source sentence and machine translation attend to each other, representations learned in such an interactive way help to identify whether a target word should be copied or be re-generated. We also find that explicitly predicting words to be copied is beneficial for improving the performance of post-editing. Experiments show that our approach achieves new state-of-the-art results on the WMT 2016 \& 2017 APE PBSMT sub-tasks.

\section{Acknowledgments}

We thank all anonymous reviewers for their valuable comments. This work is supported by the National Key R\&D Program of China (No. 2017YFB0202204), National Natural Science Foundation of China (No. 61761166008, No. 61432013), Beijing Advanced Innovation Center for Language Resources (No. TYR17002), and the NExT++ project supported by the National Research Foundation, Prime Ministers Office, Singapore under its IRC@ Singapore Funding Initiative. This research is also supported by Sogou Inc. 


\section{References}

Hanna Béchara, Yanjun Ma, and Josef van Genabith. 2011. Statistical post-editing for a statistical mt system. In MT Summit.

Rajen Chatterjee, M. Amin Farajian, Matteo Negri, Marco Turchi, Ankit Srivastava, and Santanu Pal. 2017. Multi-source neural automatic post-editing: Fbk's participation in the wmt 2017 ape shared task. In Proceedings of WMT.

Rajen Chatterjee, Matteo Negri, Raphaël Rubino, and Marco Turchi. 2018. Findings of the wmt 2018 shared task on automatic post-editing. In Proceedings of WMT.

Rajen Chatterjee, Marion Weller, Matteo Negri, and Marco Turchi. 2015. Exploring the planet of the apes: a comparative study of state-of-the-art methods for mt automatic post-editing. In Proceedings of $A C L$.

Jiatao Gu, Zhengdong Lu, Hang Li, and Victor O. K. Li. 2016. Incorporating copying mechanism in sequence-to-sequence learning. In Proceedings of $A C L$.

Tianyu He, Xu Tan, Yingce Xia, Di He, Tao Qin, Zhibo Chen, and T. M. Liu. 2018. Layer-wise coordination between encoder and decoder for neural machine translation. In Proceedings of NeurIPS.

Marcin Junczys-Dowmunt and Roman Grundkiewicz. 2016. Log-linear combinations of monolingual and bilingual neural machine translation models for automatic post-editing. In Proceedings of WMT.

Marcin Junczys-Dowmunt and Roman Grundkiewicz. 2017. An exploration of neural sequence-tosequence architectures for automatic post-editing. In Proceedings of IJCNLP.

Marcin Junczys-Dowmunt and Roman Grundkiewicz. 2018. Ms-uedin submission to the wmt2018 ape shared task: Dual-source transformer for automatic post-editing. In Proceedings of WMT.

Diederik P. Kingma and Jimmy Ba. 2014. Adam: A method for stochastic optimization. In Proceedings of ICLR.

Kevin Knight and Ishwar Chander. 1994. Automated postediting of documents. In Proceedings of AAAI.

Philipp Koehn, Hieu Hoang, Alexandra Birch, Chris Callison-Burch, Marcello Federico, Nicola Bertoldi, Brooke Cowan, Wade Shen, Christine Moran, Richard Zens, Chris Dyer, Ondrej Bojar, Alexandra Constantin, and Evan Herbst. 2007. Moses: Open source toolkit for statistical machine translation. In Proceedings of ACL.

Guillaume Lample and Alexis Conneau. 2019. Crosslingual language model pretraining. arXiv preprint arXiv:1901.07291.
Jindrich Libovický, Jindrich Helcl, Marek Tlustý, Pavel Pecina, and Ondrej Bojar. 2016. Cuni system for wmt16 automatic post-editing and multimodal translation tasks. In Proceedings of WMT.

Kathleen McKeown, Kristen Parton, Nizar Habash, Gonzalo Iglesias, and Adrià de Gispert. 2012. Can automatic post-editing make $\mathrm{mt}$ more meaningful? In Proceedings of EAMT.

Matteo Negri, Marco Turchi, Rajen Chatterjee, and Nicola Bertoldi. 2014. escape: a large-scale synthetic corpus for automatic post-editing. In Proceedings of LREC.

Jan Niehues, Eunah Cho, Thanh-Le Ha, and Alexander H. Waibel. 2016. Pre-translation for neural machine translation. In Proceedings of COLING.

Santanu Pal, Nico Herbig, Antonio Krüger, and Josef van Genabith. 2018. A transformer-based multisource automatic post-editing system. In Proceedings of WMT.

Abigail See, Peter J. Liu, and Christopher D. Manning. 2017. Get to the point: Summarization with pointergenerator networks. In Proceedings of ACL.

Rico Sennrich, Barry Haddow, and Alexandra Birch. 2015. Neural machine translation of rare words with subword units. In Proceedings of ACL.

Jaehun Shin and Jong-Hyeok Lee. 2018. Multiencoder transformer network for automatic postediting. In Proceedings of WMT.

Michel Simard, Cyril Goutte, and Pierre Isabelle. 2007. Statistical phrase-based post-editing. In Proceedings of HLT-NAACL.

Amirhossein Tebbifakhr, Ruchit Agrawal, Matteo Negri, and Marco Turchi. 2018. Multi-source transformer for automatic post-editing. In Proceedings of CLiC-it.

Inigo Jauregi Unanue, Ehsan Zare Borzeshi, and Massimo Piccardi. 2018. A shared attention mechanism for interpretation of neural automatic post-editing systems. In Proceedings of NMT@ACL.

Dusan Varis and Ondrej Bojar. 2017. Cuni system for wmt17 automatic post-editing task. In Proceedings of WMT.

Ashish Vaswani, Noam Shazeer, Niki Parmar, Jakob Uszkoreit, Llion Jones, Aidan N. Gomez, Lukasz Kaiser, and Illia Polosukhin. 2017. Attention is all you need. In Proceedings of NeurIPS.

Thuy-Trang Vu and Gholamreza Haffari. 2018. Automatic post-editing of machine translation: A neural programmer-interpreter approach. In Proceedings of EMNLP.

Robert A. Wagner and Michael J. Fischer. 1974. The string-to-string correction problem. Journal of the ACM, 21:168-173. 
Yonghui Wu, Mike Schuster, Zhifeng Chen, Quoc V. Le, Mohammad Norouzi, Wolfgang Macherey, Maxim Krikun, Yuan Cao, Qin Gao, Klaus Macherey, Jeff Klingner, Apurva Shah, Melvin Johnson, Xiaobing Liu, Lukasz Kaiser, Stephan Gouws, Yoshikiyo Kato, Taku Kudo, Hideto Kazawa, Keith Stevens, George Kurian, Nishant Patil, Wei Wang, Cliff Young, Jason Smith, Jason Riesa, Alex Rudnick, Oriol Vinyals, Gregory S. Corrado, Macduff Hughes, and Jeffrey Dean. 2016. Google's neural machine translation system: Bridging the gap between human and machine translation. arXiv preprint arXiv:1609.08144.

Baosong Yang, Zhaopeng Tu, Derek F. Wong, Fandong Meng, Lidia S. Chao, and Tong Zhang. 2018. Modeling localness for self-attention networks. In Proceedings of EMNLP.

Jiacheng Zhang, Yanzhuo Ding, Shiqi Shen, Yong Cheng, Maosong Sun, Huan-Bo Luan, and Yang Liu. 2017. Thumt: An open source toolkit for neural machine translation. arXiv preprint arXiv: 1706.06415 .

Wei Ke Zhao, Liang Wang, Kewei Shen, Ruoyu Jia, and Jingming Liu. 2019. Improving grammatical error correction via pre-training a copy-augmented architecture with unlabeled data. In Proceedings of NAACL-HLT.

Barret Zoph and Kevin Knight. 2016. Multi-source neural translation. In Proceedings of HLT-NAACL. 